\title{
Distributed Antenna System Using Sigma-Delta Intermediate-Frequency-over-Fiber for Frequency Bands above $24 \mathrm{GHz}$
}

\author{
Chia-Yi Wu, Haolin Li, Joris Van Kerrebrouck, Achim Vandierendonck, Igor Lima de Paula, Laurens Breyne, \\ Olivier Caytan, Sam Lemey, Member, IEEE, Hendrik Rogier, Senior Member, IEEE, Johan Bauwelinck, Senior \\ Member, IEEE, Piet Demeester, Fellow, IEEE, and Guy Torfs, Senior Member, IEEE
}

\begin{abstract}
The fifth generation (5G) cellular network is expected to include the millimeter wave spectrum, to increase base station density, and to employ higher-order multiple-antenna technologies. The centralized radio access network architectures combined with radio-over-fiber (RoF) links can be the key enabler to improve fronthaul networks. The sigma-delta modulated signal over fiber (SDoF) architecture has been proposed as a solution leveraging the benefits of both digitized and analog RoF. This work proposes a novel distributed antenna system using sigma-delta modulated intermediate-frequency signal over fiber (SDIFoF) links. The system has an adequately good optical bitrate efficiency and high flexibility to switch between different carrier frequencies. The SDIFoF link transmits a signal centered at a $2.5 \mathrm{GHz}$ intermediate frequency over a $100 \mathrm{~m}$ multi-mode fiber and the signal is up-converted to the radio frequency $(24-29 \mathrm{GHz})$ at the remote radio unit. An average error vector magnitude (EVM) of $6.40 \%(-23.88 \mathrm{~dB})$ is achieved over different carrier frequencies when transmitting a $300 \mathrm{MHz}$-bandwidth 64QAM OFDM signal. The system performance is demonstrated by a $2 \times 1$ multiple-input single-output system transmitting $160 \mathrm{MHz}-$ bandwidth 64-QAM OFDM signals centered at $25 \mathrm{GHz}$. Owing to transmit diversity, an average gain of $1.12 \mathrm{~dB}$ in EVM is observed. This work also evaluates the performance degradation caused by asynchronous phase noise between remote radio units. The performance shows that the proposed approach is a competitive solution for the $5 \mathrm{G}$ downlink fronthaul network for frequency bands above $24 \mathrm{GHz}$.
\end{abstract}

Index Terms-distributed antenna system, millimeter wave, multiple-input single-output, radio-over-fiber, sigma-delta modulation.

Manuscript received January 3, 2020; revised February 21, 2020; accepted February 22, 2020. This work was supported by the ERC Advanced Grant ATTO project (No.695495) and H2020 5G-PHOS project (No.761989).

C.-Y. Wu, H. Li, J. Van Kerrebrouck, A. Vandierendonck, I. Lima de Paula, O. Caytan, S. Lemey, H. Rogier, J. Bauwelinck, P. Demeester, and G. Torfs are with IDLab, Department of Information Technology, Ghent University imec, 9052 Ghent, Belgium (e-mail: chiayi.wu@ugent.be; haolin.li@ugent.be joris.vankerrebrouck@ugent.be; achim.vandierendonck@ugent.be; igor.lima depaula@ugent.be; olivier.caytan@ugent.be; sam.lemey@ugent.be; hendrik. rogier@ugent.be; johan.bauwelinck@ugent.be; piet.demeester@ugent.be; guy.torfs@ugent.be).

L. Breyne is with IDLab, Department of Information Technology, Ghent University - imec, 9052 Ghent, Belgium, and also with Photonics Research Group, Department of Information Technology, Ghent University - imec, 9052 Ghent, Belgium (e-mail: laurens.breyne@ugent.be).

Color versions of one or more of the figures in this paper are available online at http://ieeexplore.ieee.org.

Digital Object Identifier

\section{INTRODUCTION}

$\mathbf{T}$ HE fifth generation (5G) cellular network demands massive device connectivity, high data rates, and sustainable cost [1]. To meet these highly challenging demands, $5 \mathrm{G}$ is expected to include millimeter wave (mmWave) spectrum for wider signal bandwidths, to increase base station density for more network capacity, and to exploit higher-order multipleinput multiple-output (MIMO) technologies for higher wireless spectral efficiency [2]. The radio access network (RAN) must evolve to enable these technologies.

In the 4G era, the centralized RAN (C-RAN) architecture has demonstrated its advantages: lower deployment cost, easier maintenance, and higher power efficiency [3]. Various solutions have been proposed for the fronthaul interface of $5 \mathrm{G} \mathrm{C}$ RAN. Radio-over-fiber ( $\mathrm{RoF})$ technologies, including digitized radio-over-fiber (DRoF), analog radio-over-fiber (ARoF), and sigma-delta modulated signal over fiber (SDoF), are among the most convincing candidates owing to their high capacity and low latency [4]. The digitized Common Public Radio Interface (CPRI) [5], a standard that is often combined with DRoF technologies, remains the base for commercial fronthaul solutions by all major vendors until now [6]. To accommodate the 5G data traffic, enhanced CPRI (eCPRI) efficiently decreases the demanded data rate over fiber [7] at the cost of more complicated remote radio units (RRUs). For 5G frequency range 1 (FR1) $(410 \mathrm{MHz}-7.125 \mathrm{GHz})$ [8], eCPRI can be a probable solution.

For 5G frequency range 2 (FR2) (frequency bands above $24 \mathrm{GHz}$ ), there is no commercial fronthaul solution yet [6]. Plentiful publications favor ARoF [9]-[12]. ARoF can contain signals with a wide bandwidth for its good optical spectral efficiency and it is appealing when combining systems with higher-order MIMO technologies for its simple RRU architecture. However, it has strict linearity requirements on both electrical and optical devices [13].

SDoF has been proposed as a solution leveraging the benefits of DRoF (relaxed linearity requirements on both optical and electrical components) and ARoF (simple RRU architectures) [14]-[16]. We have published real-time transmission experiments of single carrier data over SDoF links for FR1 [17] and the 22.75-27.5 GHz band [18] to demonstrate the viability of the technology for future fronthaul networks. Our demo at the 45th European Conference on Optical Communi- 


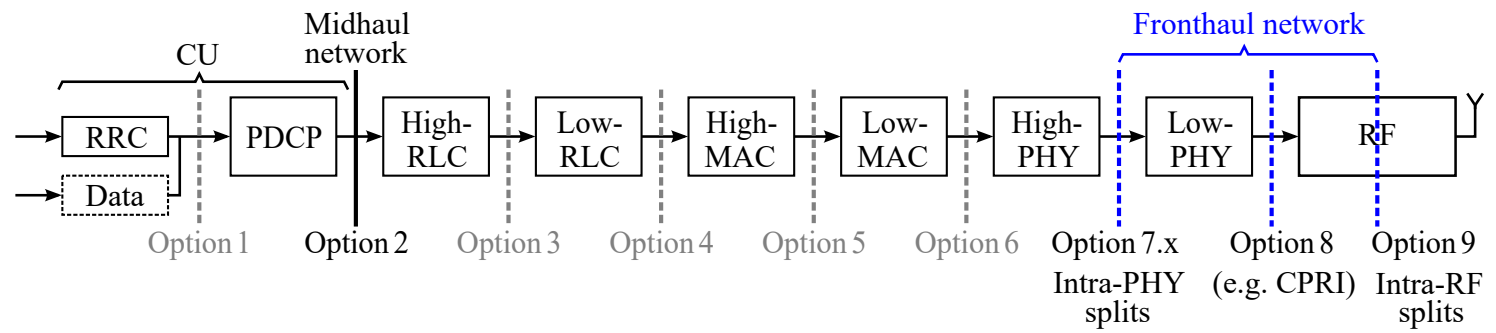

Fig. 1. Function split options [3]. (CU: central unit; RRC: radio resource control; PDCP: packet data convergence protocol layer; RLC: radio link control layer; MAC: medium access control layer; PHY: physical layer; RF: radio-frequency layer; CPRI: Common Public Radio Interface.)

RFoF (Radio-Frequency Signal over Fiber)

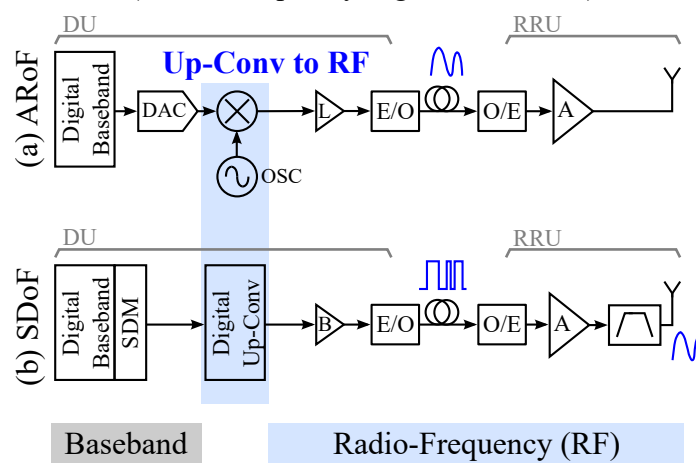

IFoF (Intermediate-Frequency Signal over Fiber)

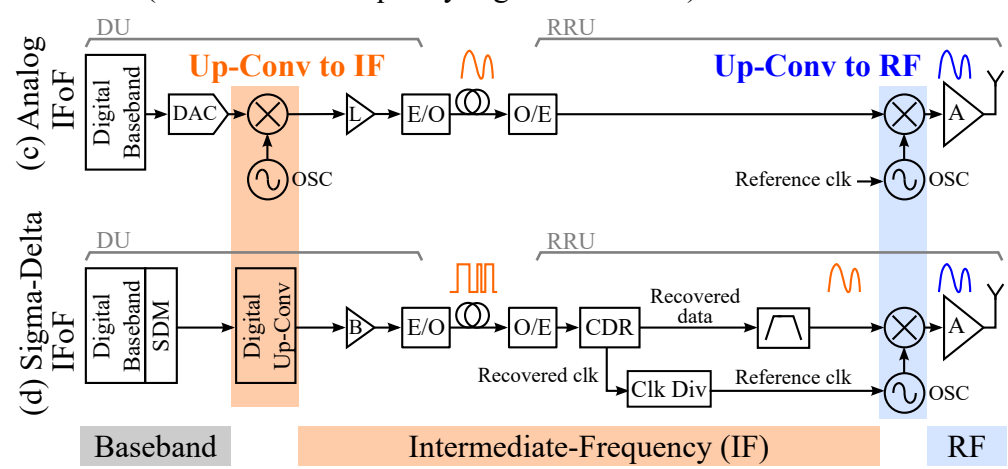

Fig. 2. (a) Analog radio-frequency signal over fiber (analog radio-over-fiber/ARoF), (b) sigma-delta modulated radio-frequency signal over fiber (SDoF), (c) Analog intermediate-frequency signal over fiber (analog IFoF), and (d) sigma-delta modulated intermediate-frequency signal over fiber (SDIFoF) link. (DU: distributed unit; RRU: remote radio unit; Up-Conv: up-conversion; E/O: electrical-to-optical; O/E: optical-to-electrical; L: linear driver; B: binary driver; A: amplifier; DAC: digital-to-analog converter; OSC: oscillator or frequency synthesizer; SDM: sigma delta modulator; CDR: clock and data recovery; Clk Div: clock divider; PLL: phase lock loop.)

cation [19] combined SDoF and MIMO techniques for FR1. The measurement results have been discussed in [20].

This paper extends our demo setup to support frequency bands above $24 \mathrm{GHz}$. We proposed a novel SDoF-based network architecture and demonstrate its performance with a $2 \times 1$ distributed multiple-input single-output (MISO) downlink transmission. The sigma-delta modulated intermediatefrequency signals centered around $2.5 \mathrm{GHz}$ are transmitted over fibers and up-converted to the desired carrier frequency at the RRUs. Each RRU exploits a clock and data recovery (CDR) module to reconstruct a clock signal from the sigmadelta modulated non-return-to-zero (NRZ) signal and use it as a reference to generate the carrier frequency. The proposed architecture achieves a higher bit-rate efficiency than our previous works [17]-[20] and offers more flexibility to switch between different carrier frequencies.

The remainder of this article is organized as follows: Section II discusses different function split options for the 5G C-RAN and radio-over-fiber technologies. Section III introduces the system architecture. In Section IV, we provide the measurement results. Section V concludes the paper.

\section{RAN FUNCTION SPLIT OPTIONS AND RADIO-OVER-FIBER TECHNOLOGIES}

For 5G C-RAN, a central unit (CU) is connected to several distributed units (DUs) via the midhaul network; each DU serves several remote radio units (RRUs) via the fronthaul network. 3GPP has decided to split the CU and DU between the packet data convergence protocol (PDCP) and radio link control (RLC) layer [3][21], as shown in Fig. 1.

The DU and RRU are traditionally split between the physical (PHY) and radio-frequency (RF) layer with CPRI applied to transmit baseband signals over fiber. When discussing the next generation fronthaul interface, both intra-PHY layer split options (Option 7.x) with eCPRI and intra-RF layer split options (Option 9) in combination with either ARoF or SDoF technologies attract intense interest. Intra-PHY split options have been comprehensively discussed in [7] and [21]. Option 9, an intra-RF split option, has first been introduced by [15].

This section focuses on intra-RF split options in which either RF or intermediate-frequency (IF) signals are transmitted over fiber depending on where the signals are up-converted to the wireless transmit carrier frequency. The RoF technologies are categorized into two groups: radio-frequency signal over fiber (RFoF) (Fig. 2a and Fig. 2b) and intermediate-frequency signal over fiber (IFoF) (Fig. 2c and Fig. 2d).

\section{A. Radio-Frequency Signal over Fiber (RFoF)}

Radio-frequency signal over fiber architectures centralize most apparatus at the DU and benefit from remarkably simple and low-power RRU architectures as illustrated in Fig. 2a and Fig. 2b. For both ARoF (Fig. 2a) and SDoF (Fig. 2b), the signals are up-converted to the radio frequency at the DU and, therefore, the synchronization of the RF carrier 

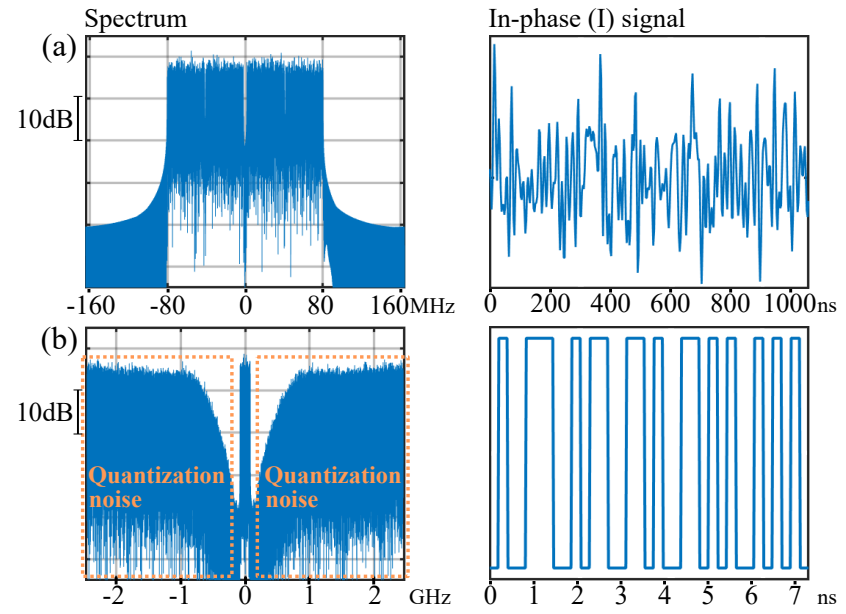

Fig. 3. (a) Spectrum and in-phase signal waveform of a $160.32 \mathrm{MHz}$ OFDM baseband signal generated by MATLAB. (b) Spectrum and in-phase signal waveform of the sigma-delta modulated (a) at $4.9152 \mathrm{GSps}$ (samples per second).

between transmitters is inherently guaranteed-one of the large challenges for distributed MIMO transmission [22]. Distributed MIMO systems using RFoF architectures suffer no performance degradation caused by the carrier asynchronism between transmitters, thus need no sophisticated synchronization algorithm [20].

In addition to the simple RRU architecture, ARoF architectures are often preferred for their high optical spectral efficiency, especially for applications with high signal bandwidths. However, they are prone to distortion and non-linearities at both the DU and the RRU side [14].

In SDoF architectures, the signals are sigma-delta modulated, i.e. oversampled and quantized to 1-bit signals. The bilevel sigma-delta modulated signal contains the original analog signal to transmit over fiber. Thus, they can be reconstructed at RRUs by simply filtering out the quantization noise which is shaped out of the band of interest as shown in Fig. 3(b). The digital characteristic of the modulated signals loosens the linearity requirements. Digital up-conversion [23] is commonly used to convert the bi-level signals to RF signals.

Despite the advantages and good performance, SDoF links are often challenged because the optical bit-rate efficiency is not always improved compared to CPRI. Two main reasons limit the bit-rate efficiency. First of all, the oversampling ratio (OSR)

$$
O S R=\frac{f_{\Sigma \Delta} / 2}{B W}
$$

of sigma-delta modulation directly affects the quality of the modulated signal; $f_{\Sigma \Delta}$ is the sample rate of the sigma-delta modulator (SDM) and $B W$ is the signal bandwidth. Secondly, when up-converting the signals digitally to the desired carrier frequency at the DU, the bit rate over fiber is consequently equal to four times the desired carrier frequency [23]. [15] uses a $960 \mathrm{MHz}$ carrier frequency and achieves a bit-rate efficiency gain of 1.85 with respect to CPRI. [20] has a lower bit-rate efficiency ( 0.43 with respect to CPRI) because the signals are up-converted to $3.5 \mathrm{GHz}$.
TABLE I

OFDM SIGNAL PARAMETERS

\begin{tabular}{llll}
\hline Parameters & Values & \\
\hline Intermediate frequency & $2.4576 \mathrm{GHz}$ & \\
Radio frequency & 24 to $29 \mathrm{GHz}$ & \\
Cyclic prefix (CP) size & $1 / 4$ & & \\
Subcarrier spacing & $320 \mathrm{KHz}$ & & \\
Data bandwidth (MHz) & 160.32 & 249.92 & 299.20 \\
$\quad N_{F F T}$ & 512 & 1024 & 1024 \\
$N_{D C}+N_{N \text { ull }}$ & 28 & 254 & 100 \\
$N_{\text {Pilots }}$ & 16 & 26 & 32 \\
Data rate per user & & & \\
$\quad$ 64-QAM (Mbps) & 718.85 & 1142.78 & 1370.11 \\
\hline
\end{tabular}

$N_{F F T}$ : number of subcarriers (FFT/IFFT size);

$N_{D C} / N_{N u l l} / N_{\text {Pilots }}$ : number of DC/null/pilot subcarriers.

For networks that do not require high bit-rate efficiency or need simple RRUs, SDoF architectures can be a good candidate. We have demonstrated an SDoF-based distributed antenna system as a possible solution for the fronthaul network deployment for the high-capacity hot-spot scenario of the $5 \mathrm{G}$ enhanced mobile broadband (eMBB) service [24]. It is also worth mentioning that sigma-delta modulated signals have higher bit error rate tolerance as evaluated in [25] and [26].

\section{B. Intermediate-Frequency Signal over Fiber (IFoF)}

Two IFoF architectures - analog IFoF and sigma-delta modulated IFoF (SDIFoF) - are illustrated in Fig. 2c and Fig. 2d respectively. The signals are up-converted to the intermediate frequency at the DU and then up-converted to the radio frequency at each RRU.

For frequency bands above $24 \mathrm{GHz}$, analog IFoF architectures can be more advantageous than RFoF ones because the signals suffer more power fading induced by fiber dispersion [27] and the high RF carrier results in the decrease of optical spectral efficiency. While compromising the low RRU complexity, analog IFoF architectures relax the hardware requirements at the DU and keep a high optical spectral efficiency [9].

Although [18] has demonstrated an all-digital SDoF link for the $22.75-27.5 \mathrm{GHz}$ band, the high bit rate over fiber is inevitable. Up-converting from an intermediate frequency unbinds the fixed relationship between the carrier frequency and the bit rate over fiber. Therefore, SDIFoF architectures have an adequately good bit-rate efficiency and the carrier frequency can be easily changed by configuring the output frequencies of the frequency synthesizers. Furthermore, when combining SDIFoF links and a distributed antenna system, no extra reference signal is needed for the up-converters. The CDR-reconstructed clock signal serves as a reference signal, as illustrated in Fig. 2d, to guarantee the carrier frequency synchronization. This is an advantage over analog IFoF links.

\section{System Architecture}

The demonstration setup consists of one distributed unit (DU), two remote radio units (RRUs), and one receiver/user. 


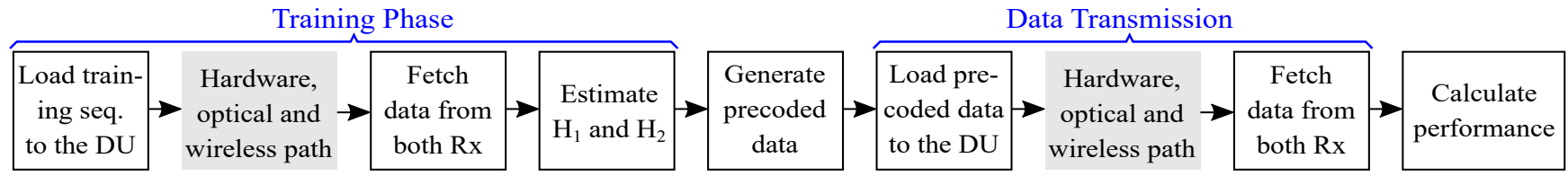

Fig. 4. Measurement workflow.

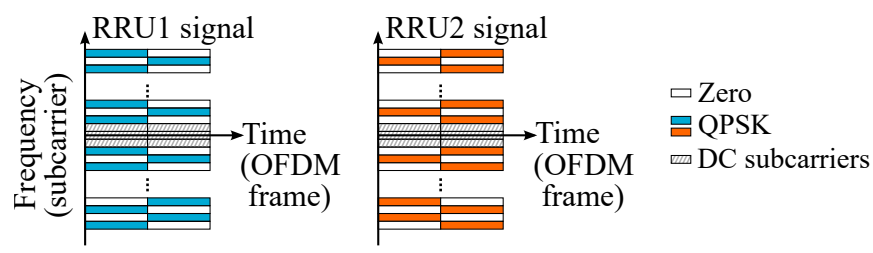

Fig. 5. Frequency-interleaved training sequence.

The DU generates two sigma-delta modulated orthogonal frequency division multiplexing (OFDM) signals centered at an intermediate frequency around $2.5 \mathrm{GHz}$ and transmits them over two multi-mode fibers. Each IF signal is independently up-converted to the radio frequency $(24-29 \mathrm{GHz})$ at a RRU before amplified and transmitted by the antenna. The two OFDM signals are precoded to interfere constructively at the receiver.

\section{A. MISO OFDM Signals}

The OFDM signal parameters used in the demonstration are summarized in Table I. For the ease of implementation and using the a priori knowledge that the experiments will be carried out in an indoor environment, the parameters are based on the IEEE802.11ac specifications [28]. It should be noted that the SDoF approach is standard agnostic, hence the employed modulation format can be easily adapted.

The sample rate of the analog front-end evaluation kit (Analog Device FMCOMMS1-EBZ) needs to be a simple fraction of $122.88 \mathrm{MHz}$. The baseband signals are sampled at $327.68 \mathrm{MSps}$ (samples per second) $(122.88 \mathrm{M} \times 8 / 3)$. To match the sample rate, the OFDM signals are generated with $320 \mathrm{KHz}$ subcarrier spacing $(\Delta f)$ such that $\Delta f \times N_{F F T}$, the OFDM bandwidth including the zero band-edge subcarriers, equals either $327.68 / 2 \mathrm{MHz}$ or $327.68 \mathrm{MHz}$. The analog-todigital converters (Analog Devices AD9643) at the receivers can sample frequencies up to $300 \mathrm{MHz}$. The $249.92 \mathrm{MHz}$ and $299.20 \mathrm{MHz}$ bandwidth signals are generated by nulling the high-frequency subcarriers of the 1024-point inverse fast Fourier transform (FFT). The data bandwidth in Table I is calculated by excluding the bandwidth occupied by band-edge zero subcarriers. Fig. 3a illustrates the spectrum and the inphase signal waveform of an OFDM baseband signal.

In our demonstration, two RRUs are employed. As such, the received baseband data on a subcarrier $R$ can be written as

$$
R=H_{1} X_{1}+H_{2} X_{2}+W
$$

where all elements in (2) are complex numbers; $H_{i}$ denotes the equivalent channel frequency response (CFR) in baseband between RRU $i$ and the receiver; $X_{i}$ is the baseband data transmitted by RRU $i ; W$ is the additive noise.

The workflow has two phases as shown in Fig. 4: the training and data transmission phase.

1) Training Phase: During this phase, frequencyinterleaved training sequences (Fig. 5) for channel estimation are transmitted; for each subcarrier, within one given OFDM frame, either RRU 1 or RRU 2 transmits QPSK data while the other one transmits zeros. First, the algorithm described in [29] is applied to estimate the carrier frequency offset (CFO). The CFRs are estimated using the least-squares channel estimation [30]. The training sequences should last at least two OFDM frames for the case with two RRUs.

The path with the lower path loss is selected as the main path. Without loss of generality, the RRU transmitting the signal via the main path is named RRU 1.

2) Data Transmission Phase: During this phase, RRU 1 transmits the original OFDM signal while RRU 2 transmits the precoded signal. The precoding guarantees the same path delay for both paths such that the signals from two RRUs combine constructively.

\section{B. SDIFoF-Based Network}

Fig. 6 shows our distributed antenna downlink system consisting of one distributed unit and two remote radio units connected by sigma-delta IFoF links. Table II lists all commercial components used in the experimental setup.

1) Distributed Unit (DU): The DU, including the SDMs and digital up-conversion to a $2.4576 \mathrm{GHz}$ intermediate frequency, is implemented on a Xilinx Virtex Ultrascale FPGA (VCU108). The MATLAB-generated OFDM baseband signals, whose in-phase (I) and quadrature (Q) signal are both 16-bit, are loaded to the DDR4 SDRAM device on the DU via the Ethernet connection, streamed to $2 \times 2$ (one I-Q pair per RRU) low-pass SDMs using a Xilinx AXI direct memory access (DMA) IP, and then modulated at $4.9152 \mathrm{GSps}$.

Second-order low-pass SDMs are chosen for this architecture to achieve a high signal-to-noise and distortion ratio. A parallel multi-stage scheme is employed to achieve the desired sample rate. The quantization noise is shaped by the low-pass SDMs to higher frequencies. Our previous work [31] describes the detailed hardware implementation of the SDM. Digital upconversion [23] translates the modulated I and Q signal (both 1-bit) to one $9.8304 \mathrm{Gbps}$ bi-level signal with a $2.4576 \mathrm{GHz}$ center frequency for each RRU. The bit rate is chosen based on the supported data-rate range of the CDRs Analog Devices ADN2917 (8.5-11.3 Gbps) and the passband frequency range of available band-pass filters in our lab $(2.3-2.6 \mathrm{GHz})$. 
Distributed Unit (DU)

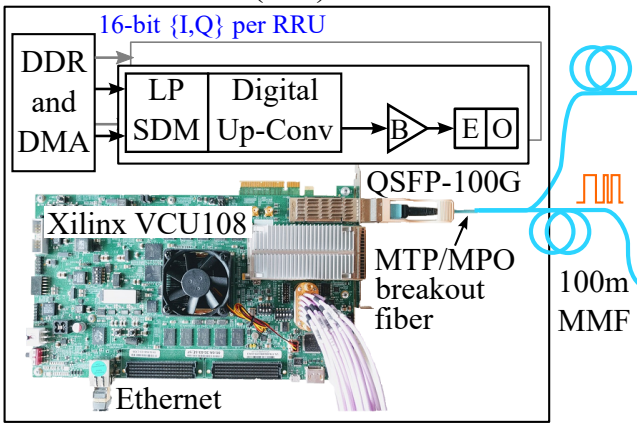

ТІІ[. Sigma-delta modulated IF signal centered around $2.5 \mathrm{GHz}$
Remote Radio Unit (RRU)

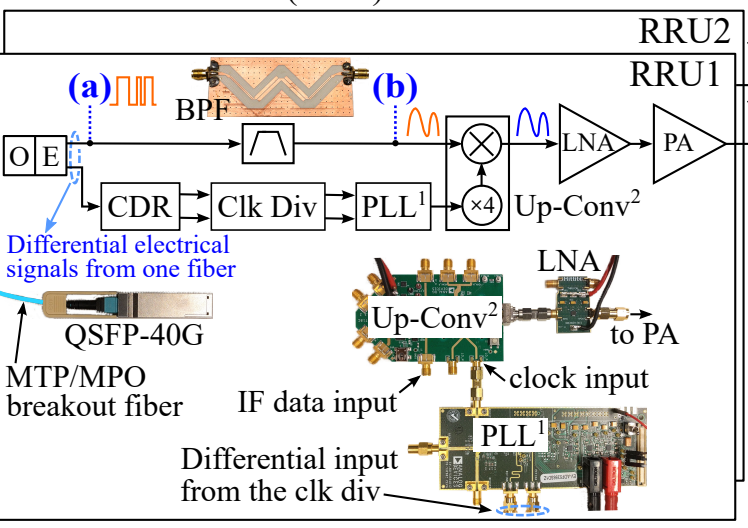

M Analog RF signal centered around $24 \mathrm{GHz} \sim 29 \mathrm{GHz}$ (a)

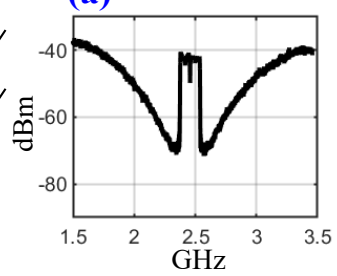

(b)

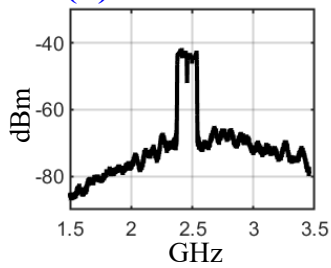

Fig. 6. System architecture. (DDR: DDR4 SDRAM device; DMA: direct memory access; LP SDM: low-pass sigma-delta modulator; B: binary driver; E-O: electrical-to-optical; O-E: optical-to-electrical; BPF: band-pass filter; LNA: low-noise amplifier; PA: power amplifier; CDR: clock and data recovery; Clk Div: clock divider; PLL: phase lock loop.) (a) Measured spectrum of the QSFP-40G output signal; (b) measured spectrum of the band-pass filter output.

${ }^{1}$ PLL-based frequency synthesizer to generate the reference input for the up-converter. ${ }^{2}$ Up-converter to convert the IF signal to RF. It has an up-mixer and a quadrupler which generates a clock signal with a frequency that is four times the frequency of the reference clock input.

The NRZ signals are converted to the optical domain using a QSFP-100G-SR4 module and transmitted over an OM4 multimode fiber (MMF). The QSFP-100G-SR4 module has four $850 \mathrm{~nm}$ VCSELs (vertical-cavity surface-emitting lasers); we use only two of them to transmit signals over two MMFs. Each MMF connects the DU to one RRU. The QSFP module supports link lengths up to $100 \mathrm{~m}$ for OM4 MMFs. The maximum optical launch power per lane is approximately $2.4 \mathrm{dBm}$. Note that the optical link lengths can be largely extended if single-mode QSFP modules and fibers are exploited [17].

2) Remote Radio Unit (RRU): The received optical signal is converted back to the electrical domain using a QSFP-40GSR4 module. The module can convert four optical signals, received from four separate fibers, to four pairs of differential electrical signals. For this setup, each RRU requires only one photo receiver out of the four. The QSFP, instead of a single photodiode, is chosen as it makes the uplink path implementation possible and allows the possibility to extend the system to more antennas in the future.

In the setup, we use one lane of the differential output for the data path and the other lane for the reference clock generation. Theoretically, CDRs can provide both reconstructed data and a reconstructed clock signal, as shown in Fig. 2d. However, the CDRs in our setup introduce too much jitter. The signalto-noise ratio (SNR) of the CDR-reconstructed data signal is worse than its original input.

Fig. 6a is the spectrum measured by an Anritsu signal analyzer (MS2692A) after the QSFP-40G-SR4 converts the received optical NRZ signal to an electrical signal. It can be seen that the quantization noise is pushed out of the band of interest. The out-of-band quantization noise is filtered by a band-pass filter (BPF), as shown in the measured spectrum (Fig. 6b). The filtered IF signal is connected to the IF data input port of the up-converter Analog Devices EVAL-ADMV1013.

At each RRU, a CDR reconstructs a clock signal from the sigma-delta modulated signal. The frequency of the re-
TABLE II

HARDWARE COMPONENTS

\begin{tabular}{|c|c|}
\hline \multicolumn{2}{|l|}{ Distributed unit (DU): } \\
\hline electrical-to-optical converter & QSFP-100G-SR4 $(850 \mathrm{~nm})$ \\
\hline \multicolumn{2}{|l|}{ Remote radio unit (RRU): } \\
\hline optical-to-electrical converter & QSFP-40G-SR4 $(850 \mathrm{~nm})$ \\
\hline $\begin{array}{l}\text { clock and data recovery } \\
\text { (CDR) }\end{array}$ & $\begin{array}{l}\text { Analog Devices ADN } 2917 \\
\text { (The CDR supports data rates be- } \\
\text { tween } 8.5 \text { Gbps and } 11.3 \mathrm{Gbps} \text {.) }\end{array}$ \\
\hline clock d & Analog Devices HMC983LP5E \\
\hline phase lock loop (PLL) & Analog Devices EVAL-ADF5356 \\
\hline
\end{tabular}

frequency synthesizer

up-converter (which has an up-mixer and a quadrupler)

low-noise amplifier (LNA)

Analog Devices EVAL-ADMV1013 (The clock input path operates from $5.4 \mathrm{GHz}$ to $10.25 \mathrm{GHz}$.)

Analog Devices HMC1040LP3CE

(The LNA operates between $24 \mathrm{GHz}$ and $43.5 \mathrm{GHz}$ and delivers $23 \mathrm{~dB}$ of small signal gain.)

power amplifier (PA)

\section{Analog Devices HMC943LP5E}

(The PA operates between $24 \mathrm{GHz}$ and $31.5 \mathrm{GHz}$ and delivers $21 \mathrm{~dB}$ of gain.)

\section{Receiver (Rx)/user:}

low-noise amplifier

band-pass filter

down-converter (which has a down-mixer and a quadrupler)

analog front-end evaluation kit

Analog Devices HMC1040LP3CE

Marki Microwave FB-2770

(Passband: $23.55 \mathrm{GHz}$ to $31.85 \mathrm{GHz}$.)

Analog Devices EVAL-ADMV1014

(The clock input path operates from $5.4 \mathrm{GHz}$ to $10.25 \mathrm{GHz}$.)

Analog Devices FMCOMMS1-EBZ

A Xilinx KC705 per user to collect data

constructed clock is half the bit rate over fiber: $4.9152 \mathrm{GHz}$. The proposed architecture uses a clock divider to generate the reference clock signal for the phase lock loop (PLL) in the frequency synthesizer. The frequency synthesizer then 
TABLE III

FREQUENCY AT THE OUTPUT OF EACH STAGE OF THE CLOCK PATH

\begin{tabular}{lcccc}
\hline Optical signal bit rate (Gbps) & 9.8304 & & \\
CDR output freq. (MHz) & $4915.2(9830.4 / 2)$ & \\
Clock divider output freq. (MHz) & $24.576(4915.2 / 200)$ & \\
& & & & \\
PLL output freq. (MHz) & 5406.72 & 5652.48 & 6144.00 & 6635.52 \\
Carrier freq. (RF) (GHz) & 24.08 & 25.07 & 27.03 & 29.00 \\
\hline
\end{tabular}

generates the reference clock signal for the up-converter.

The quadrupler in the up-converter generates a clock signal with a frequency that is four times the input clock frequency. The up-mixer modulates the IF input signal with the output clock signal of the quadrupler. The RF carrier frequencies are calculated using (3) and listed in Table III.

$$
f_{R F}=4 f_{P L L}+f_{I F}=4 f_{P L L}+2.4576 \quad(\mathrm{GHz})
$$

where $f_{P L L}$ is the frequency of the PLL output clock signal and $f_{I F}$ is the IF carrier frequency.

A low-noise amplifier (LNA) followed by a power amplifier (PA) amplifies the RF signal before feeding it to the antenna. The power measured at the PA output is about $4 \mathrm{dBm} / 160.32 \mathrm{MHz}$ for single-input single-output (SISO) cases.

Each RRU uses one in-house developed stacked air-filled substrate-integrated-waveguide (AFSIW) aperture-coupled cavity-backed patch antenna to transmit the RF signal. The compact antennas, whose dimensions are less than $5 \mathrm{~mm}$ by $-5 \mathrm{~mm}$, are implemented using the technology described in [32]. They exhibit a radiation efficiency $\left(\eta_{\text {rad }}\right)$ higher than $90 \%$ and are matched to a $50 \Omega$ impedance between $23.25 \mathrm{GHz}$ to $30.25 \mathrm{GHz}$. Within this frequency band, the radiation pattern remains stable with a minimal boresight gain of $6 \mathrm{dBi}$ and a half-power beamwidth exceeding $70^{\circ}$.

\section{Receiver and Signal Processing}

The receiver uses the same antenna as the RRUs. The antenna is first connected to an LNA. The amplified received signal is filtered by a BPF with a passband from $23.55 \mathrm{GHz}$ to $31.85 \mathrm{GHz}$ and down-converted to $2.4576 \mathrm{GHz}$ using the down-converter evaluation board Analog Devices EVAL-ADMV1014. Then, the IF signal is filtered by a BPF with a passband from $2.3 \mathrm{GHz}$ to $2.6 \mathrm{GHz}$. An analog frontend evaluation kit (Analog Device FMCOMMS1-EBZ) downconverts the IF signal to baseband and samples the baseband signal at $327.68 \mathrm{MHz}$. A Xilinx Kintex 7 FPGA (KC705) collects the data for offline signal processing using MATLAB.

The signal processing includes OFDM frame boundary detection, CFO correction, FFT, least-squares channel estimation, and QAM demodulation. The demonstration workflow illustrated in Fig. 4 is fully realized in MATLAB. Ideal channel information feedback is assumed. The CFRs estimated during the training phase are used to generate precoded data. At the receivers, after canceling the effect of the channel and the $\mathrm{CFO}$, the received data is demodulated.

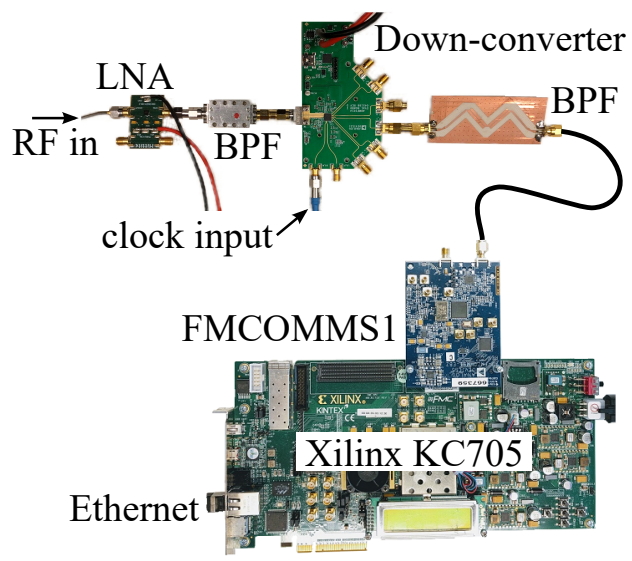

Fig. 7. Receiver. (LNA: low-noise amplifier; BPF: band-pass filter.)

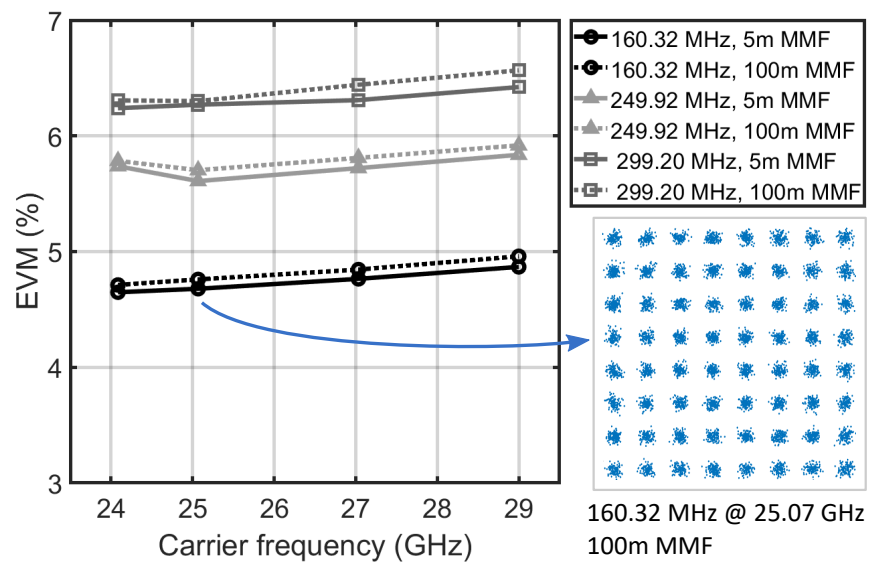

Fig. 8. SDIFoF link quality: measured EVM vs. carrier frequency (GHz). It is measured with $5 \mathrm{~m}$ (optical back-to-back) and $100 \mathrm{~m}$ multi-mode fibers (MMF) without wireless paths.

\section{Measurement Results}

In this section, first, the performance of the proposed SDIFoF link is provided by transmitting wide-bandwidth OFDM signals through the complete signal chain but without wireless paths. Second, the $2 \times 1$ MISO performance is measured by transmitting signals modulated at $25.07 \mathrm{GHz}$ in a typical office environment; the SISO performance is provided as a baseline. We also present measurement results to show that the carrier frequency synchronism is maintained in our setup. In the end, we evaluate the performance degradation due to asynchronous phase noise. The performance is presented in root-mean-square error vector magnitude (EVM) normalized to the average constellation power.

\section{A. Performance of the SDIFoF Link}

To show the quality of the SDIFoF link, we measured the performance without wireless paths. The output of the up-converter EVAL-ADMV1013 is connected directly to the input of the down-converter EVAL-ADMV1014. The output amplitude of the up-converter is properly adjusted to prevent the receiver chain from saturating. The same reference clock is provided for the up- and down-conversion using one PLL, i.e. there is no CFO. 

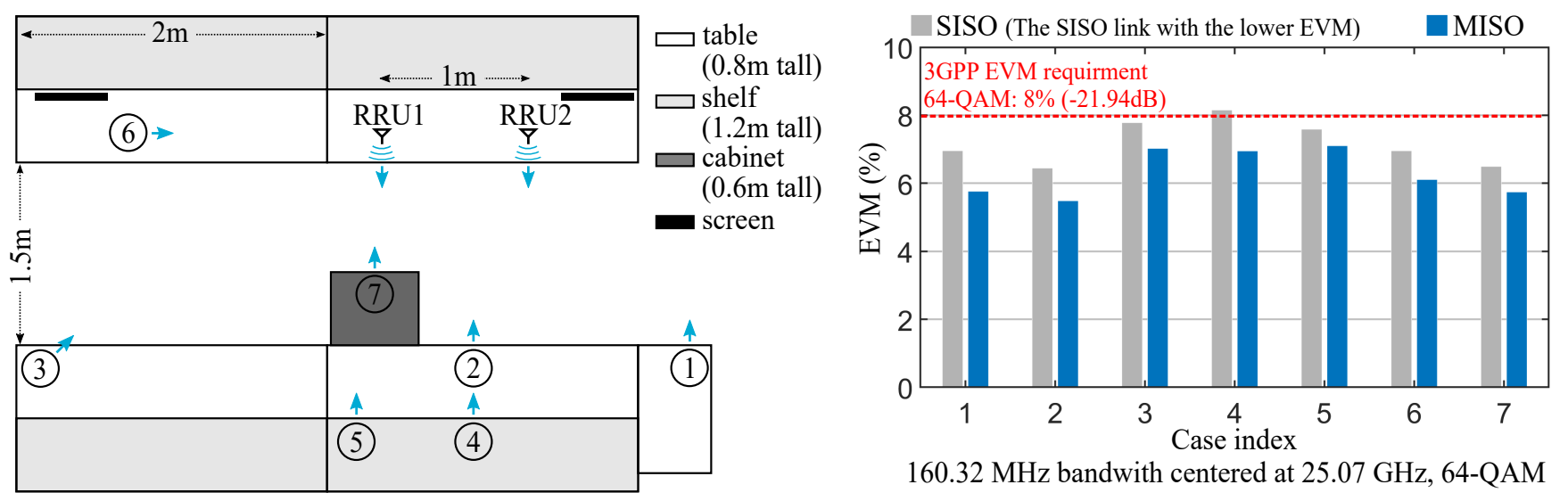

Fig. 9. Simplified layout of the measurement environment (left) and the distributed antenna system performance (right). The circled numbers denote different receiver locations. Each case in the bar chart corresponds to a receiver location.

Fig. 8 shows the EVM values versus different RF carrier frequencies. The carrier frequency can be easily adjusted by configuring the PLL output frequency while the rest of the hardware remains the same. Hence, there is no significant performance difference in terms of EVM when applying different carrier frequencies.

For optical back-to-back cases (with a $5 \mathrm{~m} \mathrm{MMF),} \mathrm{the} \mathrm{aver-}$ age EVM values are $4.74 \%(-26.48 \mathrm{~dB}), 5.73 \%(-24.84 \mathrm{~dB})$, and $6.31 \%(-24.00 \mathrm{~dB})$ for the $160.32 \mathrm{MHz}-, 249.92 \mathrm{MHz}-$, and 299.20 MHz-bandwidth OFDM signal respectively. As the signal bandwidth increases, the performance degradation is expected because of two reasons. First, the total transmitted signal power is kept the same; when the signal bandwidth increases, the power spectrum density (PSD) of the signal decreases while the noise PSD stays the same. Second, the oversampling ratio, defined as (1), decreases as the signal bandwidth increases thus resulting in a lower signal-toquantization-noise ratio.

The EVM values are higher when transmitting over a $100 \mathrm{~m}$ MMF. Nonetheless, the $6.40 \%$ EVM of the $299.20 \mathrm{MHz}-$ bandwidth OFDM signal transmitted over a $100 \mathrm{~m}$ MMF is lower than the 3GPP EVM requirements: $8 \%(-21.94 \mathrm{~dB})$ for 64-QAM [33][34].

The optical bit-rate efficiency, calculated by dividing the transmitted data bandwidth by the bit rate over fiber, is $30.44 \mathrm{MHz} / \mathrm{Gbps}(299.20 \mathrm{MHz} / 9.8304 \mathrm{Gbps})$. Using the example in [7], the bit-rate efficiency of CPRI is $27.12 \mathrm{MHz} / \mathrm{Gbps}$. The proposed SDIFoF architecture decreases the RRU complexity while maintaining the same optical bit-rate efficiency as CPRI.

\section{B. Distributed MISO Performance}

The combined optical-wireless performance is measured with a $100 \mathrm{~m}$ MMF between the DU and each RRU. Fig. 9 shows the simplified layout of the measurement environment and the measured EVM values. The two RRUs are $1 \mathrm{~m}$ away from each other.

The directions of the antennas are illustrated in the figure. The total transmit power was kept the same for the SISO and MISO cases, i.e. the transmission power of a single antenna for

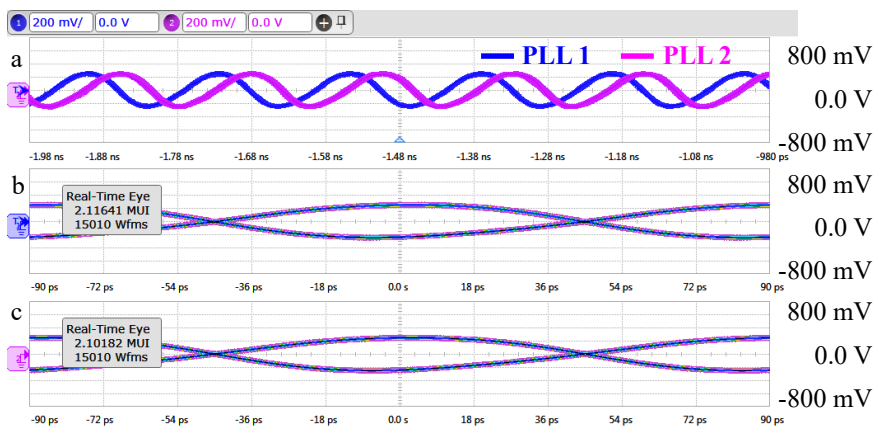

Fig. 10. PLL output signals measured by a Keysight real-time oscilloscope (DSAZ634A). (a) Captured waveform using the output of PLL 1 to trigger; (b) and (c): real-time eye diagrams of PLL 1 and PLL 2 for about 15000 frames.

SISO cases is twice the power of MISO cases. The SISO links between the receiver and both RRU 1 and RRU 2 are measured, but only the lower EVM value of the two is presented for comparison.

No common reference clock is provided to the DU, RRUs, and receiver. Fig. 10 shows the output clock signals of the PLLs measured by a Keysight real-time oscilloscope (DSAZ634A). It can be seen from the captured waveform (Fig. 10a) and the real-time eye diagrams (Fig. 10b and Fig. 10c) that the RRUs are frequency-synchronized in the setup by using the clock information contained in the sigmadelta modulated bitstreams. The CFO between the RRUs and receiver is estimated and compensated offline using MATLAB.

We measured 64-QAM OFDM signals with a $160.32 \mathrm{MHz}$ signal bandwidth. The average EVM of all SISO cases is 7.19\% $(-22.86 \mathrm{~dB})$ and $6.31 \%(-24.00 \mathrm{~dB})$ for MISO. The gain is about $1.12 \mathrm{~dB}$. For some antenna locations, it is possible to transmit higher-bandwidth signals. $160.32 \mathrm{MHz}$ is chosen for the ease of comparison so that all cases can receive 64-QAM signals.

To further evaluate the benefit of distributed antenna systems, the two RRUs are placed $2 \mathrm{~m}$ away from each other in the second measurement scenario (Fig. 11). The first three cases are selected for comparison and the performance of three measured cases is summarized in Table IV. 


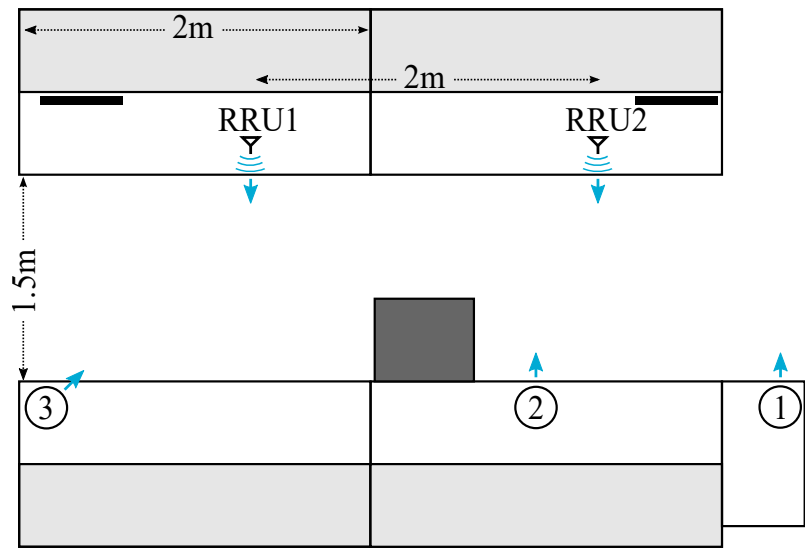

Fig. 11. Simplified layout of the measurement environment. The circled numbers denote different receiver locations.

TABLE IV

EVM COMPARISON BETWEEN TWO DIFFERENT RRU DISTANCES

\begin{tabular}{clccc}
\hline RRU distance & Case index & 1 & 2 & 3 \\
\hline \multirow{3}{*}{$1 \mathrm{~m}$} & SISO & $6.95 \%$ & $6.44 \%$ & $7.78 \%$ \\
& MISO & $5.76 \%$ & $5.48 \%$ & $7.02 \%$ \\
& Gain (dB) & 1.63 & 1.40 & 0.89 \\
\hline \multirow{3}{*}{$2 \mathrm{~m}$} & SISO & $6.50 \%$ & $6.15 \%$ & $6.44 \%$ \\
& MISO & $7.11 \%$ & $5.18 \%$ & $6.02 \%$ \\
& Gain (dB) & -0.77 & 1.35 & 0.59 \\
\hline
\end{tabular}

Case 1: When the RRU distance is $1 \mathrm{~m}$, a good gain of $1.63 \mathrm{~dB}$ owing to transmit diversity is observed. However, when RRU 1 is moved away from the receiver, the receiver is mainly served by RRU 2 . The SISO performance is better because the transmission power of RRU 2 in the SISO cases was doubled with respect to the MISO transmission.

Case 2: The receiver can receive good quality signals from RRU 1 and RRU 2 for both RRU distances, thus the performance is similar.

Case 3: When the RRU distance is $1 \mathrm{~m}$, both antennas are quite far from the receiver. A gain is observed but the EVM value is among the higher ones. When RRU 1 is moved closer to the receiver, the performance improves significantly. The transmit diversity gain is less obvious for the same reason as Case 1: the receiver is mainly served by RRU 1 and the transmission power per RRU is stronger while measuring SISO cases.

Placing the RRUs further apart-more distributed-does not necessarily lead to more transmit diversity gain, but it can provide more uniform coverage. A similar conclusion has also been derived from the single-user measurement results published in [16].

\section{Impact of Asynchronous Phase Noise}

This subsection evaluates the performance degradation due to the asynchronous phase noise introduced by the two PLLs. Note that the carrier frequencies of the two RRUs are synchronous, as shown in Fig. 10.

In Table V, the "Asynchronous RRUs" row lists the MISO performance presented in Fig. 9. The reference clock signals

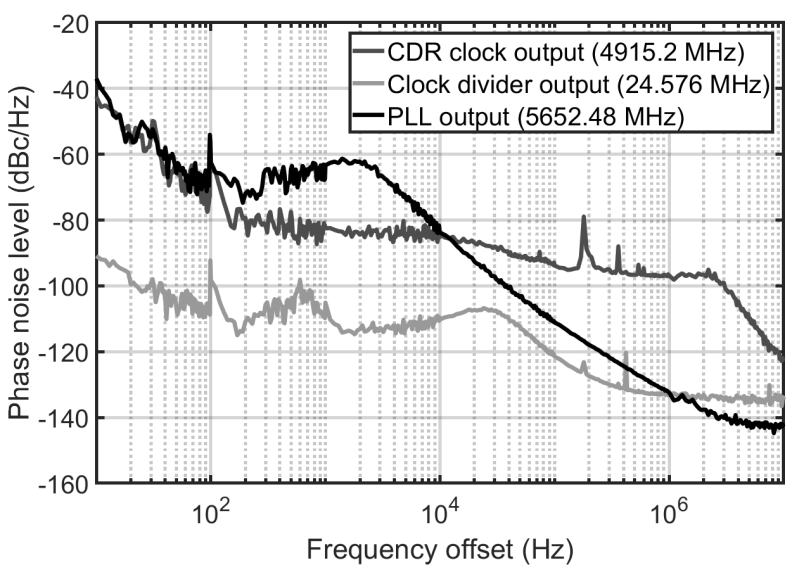

Fig. 12. Measured phase noise.

for the up-converters at two RRUs are independently generated by two PLLs, hence asynchronous phase noise is introduced. The results listed in the "Synchronous RRUs" row are measured by using the output of the PLL at RRU 1 for the upconverters of both RRUs.

Performance loss ranging from 0.35 to $0.83 \mathrm{~dB}$ is observed except for Case 6. For Case 6, the receiver is mainly served by one RRU and the signal from the other RRU is not dominating. This results in the close performance for both the synchronous and asynchronous cases.

The measured phase noise is plotted in Fig. 12. The PLLs introduce considerably large phase noise. We expect a performance improvement if better PLLs are used.

\section{CONClusion}

This work demonstrated a fully implemented $2 \times 1$ distributed MISO OFDM downlink system using real-time sigmadelta modulated intermediate-frequency signal over fiber (SDIFoF) links. The OFDM baseband signals are sigma-delta modulated and digitally up-converted to an intermediate frequency around $2.5 \mathrm{GHz}$ on an FPGA. The signals are transmitted over OM4 multi-mode fibers using a commercial QSFP module at $850 \mathrm{~nm}$. At each remote radio unit, the clock information contained in the sigma-delta modulated signal is retrieved using a clock and data recovery module. This architecture guarantees the frequency synchronism between remote radio units and requires no extra reference clock signal for synchronization.

The performance of the SDoF link satisfies the 3GPP error vector magnitude (EVM) requirements for 64-QAM (8\%): the EVM of $299.20 \mathrm{MHz}$-bandwidth OFDM signals over $100 \mathrm{~m}$ OM4 multi-mode fibers is $6.40 \%(-23.88 \mathrm{~dB})$ for different carrier frequencies ranging from $24 \mathrm{GHz}$ to $29 \mathrm{GHz}$. The same hardware implementation is used for different signal bandwidths and the carrier frequency can be easily adjusted by configuring the phase lock loops (PLLs), proving that the SDIFoF link is highly flexible. For the MISO measurements, an average gain of $1.12 \mathrm{~dB}$ owing to transmit diversity is observed despite the performance degradation due to asynchronous phase noise introduced by the independent PLLs. 
TABLE V

MISO PERFORMANCE COMPARISON BETWEEN ASYNCHRONOUS AND SYNCHRONOUS RRUS

\begin{tabular}{lccccccc}
\hline & \multicolumn{7}{c}{ Case index } \\
& 1 & 2 & 3 & 4 & 5 & 6 & 7 \\
\hline Asynchronous RRUs: & & & & & & \\
EVM in percentage (EVM in dB) & $5.76(-24.79)$ & $5.48(-25.22)$ & $7.02(-23.07)$ & $6.95(-23.16)$ & $7.10(-22.97)$ & $6.11(-24.28)$ & $5.74(-24.82)$ \\
\hline $\begin{array}{l}\text { Synchronous RRUs: } \\
\text { EVM in percentage (EVM in dB) }\end{array}$ & $5.53(-25.14)$ & $4.98(-26.05)$ & $6.71(-23.47)$ & $6.34(-23.95)$ & $6.70(-23.47)$ & $6.14(-24.23)$ & $5.47(-25.25)$ \\
\hline Performance loss (dB) & 0.35 & 0.83 & 0.4 & 0.79 & 0.5 & -0.05 & 0.43 \\
\hline
\end{tabular}

In summary, this work provides a competitive solution for the $5 \mathrm{G}$ downlink fronthaul network for frequency bands above $24 \mathrm{GHz}$.

\section{REFERENCES}

[1] A. Gupta and R. K. Jha, "A Survey of 5G Network: Architecture and Emerging Technologies," IEEE Access, vol. 3, pp. 1206-1232, Jul. 2015.

[2] J. G. Andrews, S. Buzzi, et al., "What Will 5G Be?," IEEE J. Sel. Areas Commun., vol. 32, no. 6, pp. 1065-1082, Jun. 2014.

[3] C. I, H. Li, et al., "RAN Revolution with NGFI (xhaul) for 5G," J. Lightw. Technol., vol. 36, no. 2, pp. 541-550, Jan. 2018.

[4] C. Ranaweera, E. Wong, et al., "5G C-RAN with Optical Fronthaul: an Analysis from a Deployment Perspective," J. Lightw. Technol., vol. 36, no. 11, pp. 2059-2068, Jun. 2018.

[5] "Common Public Radio Interface (CPRI) Specification V7.0," Oct. 2015. [Online] Available: http://www.cpri.info/spec.html.

[6] G. Kalfas, C. Vagionas, et al., "Next Generation Fiber-Wireless Fronthaul for 5G mmWave Networks," IEEE Commun. Mag., vol. 57, no. 3, pp. 138-144, Mar. 2019.

[7] "Common Public Radio Interface: eCPRI Interface Specification V2.0," May 2019. [Online] Available: http://www.cpri.info/spec.html.

[8] 3GPP TS 38.101-1: "New Radio (NR): User Equipment (UE) Radio Transmission and Reception; Part 1: Range 1 Standalone (Release 16) (V16.1.0)," 2019.

[9] M. Sung, J. Kim, et al., "5G Trial Services Demonstration: IFoF-Based Distributed Antenna System in $28 \mathrm{GHz}$ Millimeter-Wave Supporting Gigabit Mobile Services," J. Lightw. Technol., vol. 37, no. 14, pp. 35923601, Jul. 2019.

[10] L. Cheng, M. M. U. Gul, et al., "Coordinated Multipoint Transmissions in Millimeter-Wave Radio-over-Fiber Systems," J. Lightw. Technol., vol. 34, no. 2, pp. 653-660, Jan. 2016.

[11] U. Habib, A. E. Aighobahi, et al., "Analog Radio-Over-Fiber Supported Increased RAU Spacing for $60 \mathrm{GHz}$ Distributed MIMO Employing Spatial Diversity and Multiplexing," J. Lightw. Technol., vol. 36, no. 19, pp. 4354-4360, Oct. 2018.

[12] N. Argyris, G. Giannoulis, et al., "A 5G mmWave Fiber-Wireless IFoF Analog Mobile Fronthaul Link With up to 24-Gb/s Multiband Wireless Capacity," J. Lightw. Technol., vol. 37, no. 12, pp. 2883-2891, Jun. 2019.

[13] M. Tornatore, G.-K. Chang and G. Ellinas, "Fiber-Wireless Convergence in Next-Generation Communication Networks: Systems, Architectures, and Management," Switzerland: Springer, 2017.

[14] L. Breyne, G. Torfs, et al., "Comparison between analog radio-overfiber and sigma delta modulated radio-over-fiber," IEEE Photon. Technol. Lett., vol. 29, no. 21, pp. 1808-1811, Nov. 2017.

[15] J. Wang, Z. Jia, et al., "Delta-Sigma Modulation for Next Generation Fronthaul Interface," J. Lightw. Technol., vol. 37, no. 12, pp. 2838-2850, Jun. 2019.

[16] I. C. Sezgin, M. Dahlgren, et al. "A Low-Complexity DistributedMIMO Testbed Based on High-Speed Sigma-Delta-over-Fiber," IEEE Trans. Microw. Theory Tech., vol. 67, no. 7, pp. 2861-2872, Jul. 2019.

[17] C.-Y. Wu, H. Li, et al., "Real-Time $4 \times 3.5$ Gbps Sigma Delta Radioover-Fiber for a Low-Cost 5G C-RAN Downlink," in Proc. Eur. Conf. Opt. Commun., Rome, Italy, Sep. 2018, Paper Tu3B.1.

[18] H. Li, M. Verplaetse, et al., "Real-Time 100-GS/s Sigma-Delta Modulator for All-Digital Radio-over-Fiber Transmission," J. Lightw. Technol., vol. 38, no. 2, pp. 386-393, Jan. 2020.

[19] C.-Y. Wu, H. Li, et al., "Distributed MU-MIMO Demonstration Using FPGA-Based Sigma-Delta-over-Fiber," in Proc. Eur. Conf. Opt. Commun., Dublin, Ireland, Sep. 2019, Demo session.
[20] C.-Y. Wu, H. Li, et al., "Distributed Multi-User MIMO Transmission Using Real-Time Sigma-Delta-over-Fiber for Next Generation Fronthaul Interface," J. Lightw. Technol., vol. 38, no. 4, pp. 705-713, Feb. 2020.

[21] 3GPP TR 38.801: "Study on New Radio Access Technology: Radio Access Architecture and Interfaces (Release 14) (V14.0.0)," 2017.

[22] S. Jayaprakasam, S. K. A. Rahim, et al., "Distributed and Collaborative Beamforming in Wireless Sensor Networks: Classifications, Trends, and Research Directions," IEEE Commun. Surveys Tuts., vol. 19, no. 4, pp. 2092-2116, Fourthquarter 2017.

[23] A. Frappé, A. Flament, et al., "An All-Digital RF Signal Generator Using High-Speed $\Delta \Sigma$ Modulators," IEEE J. Solid-State Circuits, vol. 44, no. 10, pp. 2722-2732, Oct. 2009.

[24] D. Jiang and G. Liu, "An Overview of 5G Requirements," in $5 G$ Mobile Communications, Switzerland: Springer, 2017, pp. 3-26.

[25] J. Wang, Z. Jia, et al., "Delta-Sigma Digitization and Optical Coherent Transmission of DOCSIS 3.1 Signals in Hybrid Fiber Coax Networks," J. Lightw. Technol., vol. 36, no. 2, pp. 568-579, Jan. 2018.

[26] I. C. Sezgin, J. Gustavsson, et al., "Effect of VCSEL Characteristics on Ultra-High Speed Sigma-Delta-Over-Fiber Communication Links," $J$. Lightw. Technol., vol. 37, no. 9, pp. 2109-2119, May 2019.

[27] U. Gliese, S. Norskov and T. N. Nielsen, "Chromatic dispersion in fiberoptic microwave and millimeter-wave links," IEEE Trans. Microw. Theory Tech., vol. 44, no. 10, pp. 1716-1724, Oct. 1996.

[28] IEEE 802, "IEEE 802.11ac-2013," 2013.

[29] A. N. Mody and G. L. Stüber, "Synchronization for MIMO OFDM Systems," Proc. GLOBECOM, San Antonio, United States, Nov. 2001, vol. 1, pp. 509-513.

[30] J.-J. van de Beek, O. Edfors, et al., "On Channel Estimation in OFDM Systems," 1995 IEEE 45th Vehicular Technology Conference. Countdown to the Wireless Twenty-First Century, Chicago, United States, Jul. 1995, pp. 815-819 vol.2.

[31] H. Li, L. Breyne, et al., "A 21-GS/s Single-Bit Second-Order DeltaSigma Modulator for FPGAs," IEEE Trans. Circuits Syst., II, Exp. Briefs, vol. 66, no. 3, pp. 482-486, Mar. 2019.

[32] Q. Van den Brande, S. Lemey, et al., "Highly Efficient Impulse-Radio Ultra-Wideband Cavity-Backed Slot Antenna in Stacked Air-Filled Substrate Integrated Waveguide Technology," IEEE Trans. Antennas Propag., vol. 66, no. 5, pp. 2199-2209, May 2018.

[33] 3GPP TS 36.104, "Evolved Universal Terrestrial Radio Access (EUTRA): Base Station (BS) Radio Transmission and Reception (Release 16) (V16.3.0)," Sep. 2019.

[34] 3GPP TS 38.104, "New Radio (NR): Base Station (BS) Radio Transmission and Reception (Release 16) (V16.1.0)," Sep. 2019. 